https://helda.helsinki.fi

\title{
Arguments for the cognitive social sciences
}

\section{Kaidesoja, Tuukka}

2019

Kaidesoja , T , Sarkia , M \& Hyyryläinen , M 2019 , ' Arguments for the cognitive social sciences ' , Journal for the Theory of Social Behaviour, vol. 49 , no. 4 , pp. 480-498 . https://doi.org/10.1111/jtsb.122

http://hdl.handle.net/10138/310490

https://doi.org/10.1111/jtsb.12226

acceptedVersion

Downloaded from Helda, University of Helsinki institutional repository.

This is an electronic reprint of the original article.

This reprint may differ from the original in pagination and typographic detail.

Please cite the original version. 
This is the penultimate draft of the paper published in Early View site of Journal for the Theory of Social Behaviour. https://doi.org/10.1111/jtsb.12226

\section{Arguments for the cognitive social sciences}

Tuukka Kaidesoja, Matti Sarkia \& Mikko Hyyryläinen

\section{Introduction}

The overlap between the cognitive and social sciences has significantly increased in recent decades. New disciplines and research programs have arisen and expanded at the intersection of these two types of sciences. They include cognitive sociology, political psychology, behavioral economics and new research programs in cognitive anthropology. However, not all social scientists have been persuaded that the social sciences should be integrated with the cognitive sciences. Some of them are indifferent to these new integrative disciplines and research programs, assuming that they are not relevant to their research practices. Other social scientists consider them as overly reductionist and, thereby, as a threat to the identity of their disciplines. As a response, cognitive social scientists have provided arguments to convince all social scientists about the benefits of integrating the social sciences with the cognitive sciences. In this article, we analyze and evaluate these arguments for the cognitive social sciences.

The cognitive social sciences can be defined as disciplines and research programs that aim to integrate the social sciences (e.g. sociology, anthropology, political science and economics) with the cognitive sciences (e.g. cognitive psychology, cognitive neuroscience and cognitive science). We understand interdisciplinary integration as an umbrella term that includes different ways of bringing scientific disciplines together and we separate different arguments for the cognitive social sciences on the basis of their underlying ideas about interdisciplinary integration. Although all cognitive social sciences focus on the cognitive aspects of social phenomena and relate themselves to the cognitive sciences, we do not require that the concept of cognition is defined in the same way in these integrative disciplines and research programs, nor do we require that they use similar research designs and methods. 
More precisely, our aims are to reconstruct four arguments for the cognitive social sciences by specifying their premises, inferential structures and conclusions and to evaluate these arguments by analyzing their presuppositions, the plausibility of their premises, the soundness of their inferences and potential conceptual ambiguities. We focus on the arguments that seek to justify the need of the cognitive social sciences for social scientists but we do not discuss in detail the results of different research programs of cognitive social scientists. The arguments include deep differences regarding how the integration between the social and cognitive sciences is understood, resulting in different visions as to how the cognitive social sciences might look like. They do not, however, exhaust the list of arguments that have been provided for the cognitive social sciences.

Although we are more interested in analyzing the arguments for the cognitive social sciences than mapping differences between them, different types of arguments reflect differences in cognitive social scientists' presuppositions about the objects and methods of their research. Hence, in our analysis and evaluation of these arguments, we pay special attention to their ontological, methodological and theoretical presuppositions. Ontological presuppositions concern the nature and relations of cognitive and social phenomena. Methodological presuppositions deal with the preferable methods and explanatory strategies in the empirical studies of social phenomena. Theoretical presuppositions relate to the nature of acceptable explanatory theories and models about social phenomena. Since these presuppositions often remain implicit, one of our tasks is to identify and explicate them. ${ }^{1}$

The following four sections are organized such that we first provide a concrete example of the argument under analysis. Although we have chosen our examples from the programmatic articles and books of cognitive social scientists, we admit that a single text may include more than one type of argument. We have selected our exemplary texts such that the type of argument that they illustrate is dominant in them. Then we reconstruct the structure of the argument in general terms. Each of these four sections ends with a brief discussion of the presuppositions of the argument under analysis and a critical

${ }^{1}$ Hence, we agree with Gabe Ignatow (2014) and Omar Lizardo et al. (2019), who argue that cognitive and cultural sociologists should clarify their positions in relation to the key debates in philosophy of (social) science, and with Stephen Turner (2018), who emphasizes the importance of understanding the complexity of the philosophical issues that are raised by the idea of cognitive social science. 
evaluation of its strengths and weaknesses. In the final discussion, we compare different types of arguments and identify the most compelling reasons why the cognitive social sciences are needed.

\section{Argument from explanatory grounding}

One of the pioneers of the cognitive social sciences, Ron Sun, begins his introduction to the book, Grounding Social Sciences in Cognitive Sciences, by claiming that cognitive explanations of social phenomena are often missing from the social sciences (Sun, 2012, p. 3). By cognitive explanation he means explanations that are based on the knowledge produced by the cognitive sciences. Then he argues that because "the cognitive sciences [...] have made tremendous strides in recent decades" (p. 4) and because "minds [...] are the basis of social processes and phenomena" (p. 5), "the cognitive sciences may serve as a basis for the social sciences, in much the same way that physics provides grounding for chemistry or quantum mechanics provides grounding for classical mechanics" (p. 5). Hence, according to Sun, "taking cognition-psychology into serious consideration would be a reasonable step in trying to reach an in-depth, fundamental understanding of social phenomena" (p. 5).

Sun (2012) provides many examples in order to support his argument. For instance, he discusses explanatory studies on the influence of cognitive universals on various social and cultural phenomena, such as religion and language, and his own work on the CLARION cognitive architecture. The CLARION architecture draws on cognitive science and computational modeling to develop a comprehensive, empirically informed, and modularly structured architecture of the human mind that can be used to model various cognitive and social phenomena (e.g. Sun, 2017). Hence, according to Sun's argument, cognitive social sciences are worth pursuing since they provide explanatory grounding for the social sciences and, thereby, deepen our understanding of social phenomena.

Here is our reconstruction of Sun's argument from explanatory grounding:

1) Most social scientists do not currently make use of the knowledge produced in the cognitive sciences when they explain social phenomena.

2) Cognitive processes are the ontological basis of social processes.

3) Explanations in the cognitive sciences are deeper than explanations in the social sciences because they bottom out in cognitive processes. 
4) If social scientists ground their explanations in the cognitive sciences, their explanations for social phenomena would become deeper than they are at present.

5) Conclusion: the social sciences should be grounded in the cognitive sciences.

It is important to recognize that Sun's argument presupposes that the explanatory grounding relation between the cognitive and social sciences that is asymmetrical. This means that if the social sciences are grounded in the cognitive sciences, then the cognitive sciences cannot be grounded in the social sciences.

Key premises 2 and 3 rest on the requirement that scientific explanations should reflect the ontological order of reality such that explanations of higher-level processes should be explained by the models that represent their lower-level component processes that form the ontological basis of the higher-level processes. Sun's (2012) ontological views that support this requirement are based on a (nonreductionist version of the) physicalist ontology or an emergent materialist ontology. The key assumption of this ontology is that higher-level social processes are dependent on but not reducible to the lower-level cognitive processes. ${ }^{2}$ Since Sun assumes that cognitive sciences study cognitive processes that are ontologically more fundamental than social processes studied in the social sciences, he expects that the cognitive sciences are capable of providing deeper explanations for social processes than those currently provided in the social sciences. He does not claim, however, that these cognitive explanations would explain social processes away (e.g. by means of ontologically reducing them to cognitive processes or eliminating them from scientific ontology). In other words, the idea of explanatory grounding of the social sciences in the cognitive sciences is compatible with the assumption that social processes have weakly emergent properties that can be mechanistically explained.

To what extent does the argument from explanatory grounding provide compelling reasons that should convince social scientists for the need of the cognitive social sciences? It seems that most social

\footnotetext{
${ }^{2}$ In metaphysics, the notion of supervenience is often used to elucidate the dependence relations between different level entities. The standard definition of supervenience requires that there can be no change in B-properties (the supervenient facts) without a corresponding change in A-properties (the supervenience base) (Kim, 2005). Nevertheless, more substantive way to clarify this relation is use the concept of emergence. For example, William Wimsatt's (1997) notion of emergence as a failure of aggregativity of system-level properties is interesting in this respect. It is a weak concept of emergence in the sense that, in contrast to the strong concepts of emergence, it does not deny that emergent properties can be mechanistically explained.
} 
scientists would probably agree with Sun's observation that only a few social scientists presently rely on the cognitive sciences when they explain social phenomena. Nevertheless, one must accept conclusion 5 only if one accepts premises 2, 3 and 4 as true. Not all social scientists are convinced of the truth of these premises. We will review some reasons that they might use to question them.

First of all, these premises imply that the influence of the cognitive sciences on the social sciences is unidirectional, since cognitive sciences are capable of providing deeper explanations to social processes than social sciences. Nevertheless, this idea of asymmetrical explanatory grounding may pose unnecessary constraints for the development of the cognitive sciences. We do not see any good a priori reasons to exclude the possibility that the social sciences might have something useful to offer to those parts of the cognitive sciences that address the cognitive aspects of social phenomena (even if one accepts that the social sciences study ontologically "less fundamental" processes than the cognitive sciences). Indeed, also Sun (e.g. 2012, pp. 18-23) seems to admit this. A proponent of the argument from explanatory grounding could try to cope with the previous counter-claim by granting that social sciences may be useful when it comes to providing accurate descriptions of social phenomena while demanding that cognitive sciences are needed to provide deep explanations to these phenomena (we will come back to this view below).

Second, one way to motivate premises 2 and 3 is to assume that the human mind studied in the cognitive sciences is composed of the evolved modules that are, at least to a large extent, functionally specified, domain-specific, innate and essentially the same in all "normal adults" (e.g. Barkow, Cosmides \& Tooby, 1992; Pinker, 2002; Sperber, 1996, Chapter 6). ${ }^{3}$ In this view, cognitive explanations for social phenomena are ontologically deeper than social scientific explanations precisely because the former explain social phenomena in terms of mental modules that are triggered in our modern environments that are partially different from those Pleistocene environments in which these modules originally evolved. These interacting mental modules of individuals are thereby assumed to provide the ontological basis for social processes. Also Sun's (e.g. 2012, pp. 7, 11-14, 2017) CLARION-architecture

\footnotetext{
${ }^{3}$ We recognize that the notions of mental module and innateness are both used in variety of different ways in the cognitive scientific literature, but we refrain from discussing these differences, because those cognitive social scientists who advocate the modular view of human mind often use these concepts without specifying their meanings. In addition, our arguments apply to the most popular variations of these concepts.
} 
assumes that human mind is modular and that all humans have primary drives, but he does not seem to subscribe to the strongest versions of the so called "massive modularity theses" of the nativist evolutionary psychologists.

Nevertheless, there is a growing number of cognitive scientists and philosophers of mind who reject the assumption that human minds are best understood in terms of the functionally specified modules whose development is (more or less) insensitive to the differences in the species-typical developmental environments of children and whose operations are not dependent on the broader material and social environments of embodied human action (e.g. Clark, 1997; Donald, 2001; Sterelny, 2003, 2012; Buller, 2005; Franks, 2012; Karmiloff-Smith, 2015). Hence, at least the assumption that human mind is massively modular is highly controversial although there may be weaker versions of modularity that are less problematic.

Despite the fact that some versions of the argument from explanatory grounding presuppose that human mind has a modular architecture that is largely innate, this assumption might not be necessary for this type of argument. But if we give up the modularity assumption, then we should acknowledge that the best explanations of some cognitive processes and capacities might include knowledge produced by the social sciences as they key elements. For example, if we want to explain how children learn to read and write, then instruction and social learning in particular types of material, institutional and symbolic environments would be among the key explanatory factors in addition to the cognitive scientific knowledge about those cognitive processes that take place in mind. One reason for this is that human children cannot have any innate mental modules for reading and writing due to the fact that there have not been enough time for such modules to evolve through natural selection. ${ }^{4}$ These considerations suggest that premise 3 should be modified to take into account the possibility of the two-way traffic between the cognitive and social sciences if we give up the assumption that human mind is composed of innate mental modules.

\footnotetext{
${ }^{4}$ A defender of the modular view might argue that the lack of evolved mental modules for reading and writing as such does exclude the possibility that reading and writing skills may be understood in terms of the reconfiguration of innate mental modules for new purposes. Although this is possible, it should be emphasized here that in order to understand how this reconfiguration takes place one much study processes of social learning and instruction in specific material and symbolic environments.
} 
Finally, due to the facts that abstract ontologies-such as emergent materialism-are difficult to evaluate empirically and that Sun (2012) also supports premise 4 by reviewing cognitive scientific studies and theories that have been used to deepen our understanding of some social phenomena, the strength of his argument partly depends on the quality of these examples. Although this is not right place to analyze them in detail, it should be noted that a potential pitfall of this type of examples is that they may redefine the social phenomena under study in a way that many social scientists find unacceptable. For example, Pascal Boyer (2001) has argued that many religious phenomena can be best explained in terms of the operation of a hyperactive agency-detection module and the predisposition to remember "minimally counterintuitive representations". Even if this provides a plausible cognitive explanation why religious beliefs with a specific type of content are highly contagious (we will not attempt to evaluate its scientific merits here), there are surely many institutional and cultural aspects of religion that remain unexplained, concerning religious doctrines, rituals, practices and organizations in a specific religious groups and their differences between different groups and times.

Nevertheless, this argument that highlights the variability of social phenomena can be countered by claiming that social scientists have partially misidentified the social phenomena that require explanations by uncritically relying on our everyday accounts of these phenomena. Insofar as cognitive social scientists aim at providing scientific explanations for social phenomena (in contrast to more or less local interpretations), they should re-conceptualize their explanandum in a way that takes into account the cognitive scientific knowledge about universal and domain-specific mental modules through which humans form, transform, store and transmit representations (e.g. Sperber, 1996).

It is important to recognize that this counter-argument is plausible only if we accept that human mind has a modular architecture that is largely innate. As discussed above, this assumption is highly controversial among cognitive scientists and philosophers of mind, not to mention its contentiousness among social scientists. Also the distinction between the scientific and interpretive methods, assumed in the above claim, is not accepted by all social scientists. Not even all cognitive social scientists would accept it, since some of them count interpretive (or qualitative) methods as scientific methods in addition to experimental, statistical and simulation methods (see Hutchins, 1995; Zerubavel, 1997; Bloch, 2012). Hence, we suggest that it should be judged in a case-by-case manner whether reconceptualizations of this kind are reasonable in the light of the aims of social scientific research. 
Sun (2012) has not been the only cognitive social scientist who has appealed to the need for explanatory grounding of the social sciences in the cognitive sciences. Similar arguments have been presented by the advocates of the nativist program of evolutionary psychology (e.g. Barkow et al., 1992). For example, Steven Pinker's (2002) attack against what he calls "the standard social science model", and his argument for the nativist version of evolutionary psychology includes an attempt to ground social sciences in the cognitive sciences, although there are slight differences between Pinker's notion of massively modular mind and that of Sun's (e.g. 2017).

\section{Argument from theoretical unification}

Another argument for the integration between the social and cognitive sciences is provided by Herbert Gintis who emphasizes the importance of theoretical unification. He describes a set of disciplines as unified "if they are consistent, so that in cases where two disciplines deal with the same phenomena, their models are equivalent and synergic, each discipline being substantively enriched by the scientific content of the others" (Gintis, 2004, p. 37). Gintis $(2007$, p. 1) says that the unity of the behavioral sciences requires that they have "a common underlying model, enriched in different ways to meet the particular needs of each discipline." The behavioral sciences, which include economics, anthropology, sociology, psychology, political science as well as biological research on human and animal behavior, are not unified in this way even though they all deal with the phenomena of decision-making and strategic interaction (Gintis, 2012, p. 415). Gintis contrasts the disunified array of the behavioral sciences with the more mature state of physics, chemistry and astronomy, which "achieved unity with the development of quantum mechanics, elementary particle and solid-state physics, and the big bang model of the universe" (Gintis, 2004, p. 37).

Gintis (2012, p. 415) traces the disunified state of the behavioral sciences to the three incompatible models of human behavior that they employ: biological, economic and sociological. The biological model "is that of the fitness maximizer, who is the product of a long process of Darwinian evolution" (p. 415). The sociological model describes "the pliant individual who internalizes the norms and values

of society and behaves according to the dictates of the social roles he occupies" (p. 415). The economic model is based on "rational choice theory, which takes the individual as maximizing a self-regarding preference subject to an unanalyzed and pre-given set of beliefs, called subjective priors" (p. 415). 
Gintis (2012, p. 415) contends that "all three are flawed, but can be modified to produce a unified framework for modeling choice and strategic interaction for all of the behavioral sciences through a considered appreciation of the evolved nature of human psychology" (see also Gintis, 2009).

Gintis (2012) draws on five general principles in order to unify the behavioral sciences. First, there is gene-culture coevolution, according to which "human cognitive, affective, and moral capacities are the product of an evolutionary dynamic involving the interaction of genes and culture" (p. 419). Second, there is the socio-cultural theory of norms, which "includes fundamental insights from sociology that apply to all forms of human social organization, from hunter-gatherer to advanced technological societies" (p. 416). Third, there is game theory as the general theory of "strategic interaction, in which the behavior of agents is derived by assuming that each is choosing a best response to the actions of other agents" (p. 428). Fourth, there is the rational actor model, which provides partial foundations for behavioral, epistemic and evolutionary game theory by assuming that individuals have consistent preferences which "can be represented by a numerical function [....] which the individual maximizes subject to his subjective beliefs" (p. 424). In contrast to its traditional version, Gintis' (2012, pp. 417, 423-426; also Gintis, 2007, 2009) updated version of the rational actor model relaxes the assumptions that rational actors must be perfectly informed and that their preferences must be self-regarding. Fifth, there is complexity theory which "is needed because human society is a complex adaptive system with emergent properties that cannot now, and perhaps never will be, explained starting with more basic units of analysis" (Gintis, 2012, p. 418).

Although he lists five principles for unifying the behavioral sciences, the third and the fourth principle are the main elements of the unified modeling framework that Gintis $(2007 a, 2009,2012)$ proposes for those areas of the behavioral sciences that overlap with each other. He states that "[t]he rational actor model is the single most important analytical construct in the behavioral sciences operating at the level of individual" (Gintis, 2012, p. 417) and that "[g]ame theory is a general lexicon that applies to all life forms" (Gintis, 2009, p. 45) and, thereby, "fosters a unified analytical framework available to all behavioral sciences" (Gintis, 2009, p. 45). Complexity theory is left out from this modeling framework, but this omission is not surprising given the fact that the fifth principle was added to Gintis' original principles as a response to his critics (see Gintis, 2007b, p. 45). For this reason, it is only loosely connected to his other principles. He also added the socio-cultural theory of norms later to the list of 
unifying principles since it is missing from the list of principle provided in Gintis (2007b, p. 45). Gintis (e.g. 2012, p. 432-433) conceptualizes norms in terms of the updated rational actor model and game theory, meaning that the second principle is subordinated to the third and the fourth principle.

Our reconstruction of Gintis's argument from theoretical unification is as follows:

1) Scientific disciplines that study the same domain of phenomena should be conceptually and theoretically unified with one another.

2) The behavioral sciences all study the same domain of phenomena, which have to do with the decision-making and strategic interaction.

3) Hence, the behavioral sciences ought to be unified with one another.

4) Unification of the behavioral sciences requires a unified framework for modeling decisionmaking and strategic interaction in a way that takes into account the contributions of different behavioral sciences.

5) Conclusion: The rational actor model and game theory, when modified to take into account the evolved nature of human psychology and the socio-cultural theory of norms, provide a unified modeling framework for the behavioral sciences.

Premises 1 and 3 may at first be regarded as obvious, if one takes the comparison that Gintis draws between the currently disunified state of the behavioral sciences and the more unified state of the mature natural sciences (i.e. physics, chemistry and astronomy) seriously. However, many philosophers of science, such as John Dupré (1993) and Nancy Cartwright (1999), have argued that even "mature natural sciences" are not theoretically nor ontologically as unified as it may seem at first sight. Moreover, social epistemologists have argued that a diversity of perspectives on the world is essential for scientific progress both in the natural sciences and in the social sciences (e.g. Weisberg \& Muldoon, 2009). There are also additional reasons to resist the conclusion that the behavioral sciences (including the social sciences) ought to be theoretically unified with one another, even if one accepts this claim for the mature natural sciences. For example, it has been argued that theories in the social sciences as well as in some areas of biology are essentially value-laden, and may involve conflicting power relations and political ideologies, and therefore a diversity of different points of view is essential for the these sciences (Longino, 1990, 2013). These reasons indicate that we should treat Gintis's unificationist 
agenda with caution, regardless of whether we take the behavioral sciences to be fundamentally different from the mature natural sciences with respect to their methods or objects of study.

Premise 2 should be read with the understanding that most research programs in the behavioral sciences concern some specific domain in which human behavior and interaction takes place-for example, the family, the workplace, the markets or the tribe-rather than the social world as a whole. The piecemeal character of social scientific research practice does not go entirely unnoticed by Gintis insofar as his aim is "to render models of human behavior from different disciplines compatible where they overlap" (Gintis, 2012, p. 416). Nevertheless, in addition to this modest account of unification in terms of compatibility between overlapping disciplines, which does not seem to require a unified modeling framework, Gintis presents stronger claims regarding the roles of rational actor model and game theory in the theoretical unification of the behavioral sciences that are described in premise 4.

Gintis' argument from theoretical unification is likely to raise the charge of economics imperialism (see Mäki, 2009) among social scientists, due to the central role that the rational actor model plays in his unified modeling framework and his principles for unifying the behavioral sciences. Indeed, four of the five principles that Gintis proposes are at least partly parasitical on the rational actor model. The axioms of rational choice provide the foundation for both game theory and the socio-cultural theory of norms, which Gintis (2012, p. 432) understands through the game-theoretic notion of correlated equilibrium. Moreover, gene-culture evolution is applied by way of evolutionary game theory, and complexity theory seems to play a role only as a leftover category that is there to incorporate whatever the rational actor model and game theory cannot explain, as the social world may include "emergent properties cannot be derived analytically from lower-level constructs" (Gintis, 2012, p. 416). Hence, although he discusses many topics in his papers, the unifying work in Gintis' argument from unification is done by the revised versions of rational actor model and game theory, both of which have been most influential in the neoclassical economics. The central role that they play in Gintis's unificationist agenda for the behavioral sciences is unlikely to make it appeal to many social scientists (but see Hechter \& Kanazawa, 1997), who may perceive it as a thinly veiled attempt at forcing all social sciences to succumb under the imperialist rule of economics. 
The rational actor model and game theory, combined with the relatively speculative use of some of the evolutionary principles that Gintis proposes, also raise concerns about their compatibility with empirical results of the cognitive sciences and the potential untestability of his axiomatic assumptions (e.g. Turner, 2018, pp. 31-35). Gintis (2007a, 2007b, 2009) anticipates these worries and aims to show that all cognitive scientists, psychologists and behavioral economists, who reject the rational actor model and game theory, are wrong ${ }^{5}$ and that this model can be supported by neuroscientific evidence. Nevertheless, the neuroscientific evidence that Gintis (e.g. 2007b, p. 5) provides is controversial (see Fumagalli, 2013). Many of Gintis' (e.g. 2007a, pp. 4, 9-15) answers to those critiques that cognitive scientists and psychologists have raised against the assumptions of rational actor model are question begging in the sense that he tends to explain away empirical evidence that questions the rational actor model by claiming that it results from "performance errors" of individuals that are not supposed to question their rationality that he tends to identify with the axiomatic assumption of preference consistency. In addition, many philosophers of science (e.g. Buller, 2005; Dupré, 2001) have also criticized evolutionary psychological accounts of innate or evolved human nature that is assumed in Gintis (e.g. 2007a, pp. 15-16) arguments.

For these reasons, it is not at all clear whether Gintis' principles and assumptions form an internally coherent whole and whether they are compatible with the findings of the cognitive sciences. Since he provides few examples of where his unified modelling framework for the behavioral sciences have been successfully applied to unify social scientific theories, it is also ultimately left to the reader to figure out whether it is appropriate for the purposes of integrating the social sciences with the cognitive sciences.

A very different and more implicit example of the argument from theoretical unification can be found in discursive psychology (e.g. Edwards \& Potter, 1992). The key idea of this research program is to argue that, instead of focusing on mental states and cognitive processes of individuals, psychological phenomena should be studied as social or linguistic constructions that people use to accomplish social

\footnotetext{
${ }^{5}$ Gintis, for example, defends behavioral game theory by claiming that "[b]ecause one cannot do behavioral game theory, by which I mean the application of game theory to the experimental study of human behavior, without assuming preference consistency, we must accept this axiom to avoid the analytical weaknesses of the behavioral disciplines that reject the BPC model, including psychology, anthropology, and sociology" (Gintis, 2009, p. 2). Letters BPC refer to Beliefs, Preferences and Constraints and the BPC model is another name that Gintis' gives to his revised version of the rational actor model. The previous claim boils down to a view that unified modeling frameworks are better than other explanatory approaches in the social and cognitive sciences.
} 
actions in specific situations. Discursive psychologists are agnostic about the existence of mental states apart from the uses of mental and cognitive terms in everyday discourses. Accordingly, they suggest that the proper method to study psychological phenomena of this kind is to use discourse analysis. If Gintis's argument is an instance of economics imperialism, then discursive psychology may be understood as an instance of sociological imperialism since it uses sociological theories and methods to unify psychology and sociology. One problem with discursive psychology is that it refuses to make a distinction between our ways of speaking about cognitive processes and the cognitive processes and mechanisms that actually generate our actions and interactions. Thereby, it ends up rejecting nearly all cognitive scientific research.

\section{Argument from constraints}

On the first page of his Anthropology and the Cognitive Challenge, Maurice Bloch (2012) states that "cognitive issues are not on the periphery of such social sciences as anthropology, history or sociology" (p. 1). Instead of being peripheral concerns, cognitive issues "are relevant and helpful for the most central and familiar topics which, among others, cultural and social anthropologist deal with" (p. 1). According to Bloch (p. 7), anthropologists and other social scientists end up doing cognitive anthropology "as soon as they claim to represent knowledge of those they study, as soon as they try to explain actions of people in terms of that knowledge, as soon as they warn general public, or each other, of the dangers of ethnocentrism, as soon as they discuss the extent, or the limits, of cultural variability." In his view, then, social scientists cannot escape from making assumptions about the cognition of their research subjects. The question is: where do these assumptions come from?

Bloch's (2012, p. 8) answer is that the cognitive assumptions of anthropologist and other social scientists are often based on "the hazy cognitive theories of folk wisdom, their own and those of the people they study". Then he points out that one of the main contributions of the cognitive sciences has been to question the folk theories of this kind (pp. 8-9). Many social scientist, however, are not only ignorant of this fact but also fail to distinguish between (i) what their research subjects say about their cognition when they are interviewed or observed by using participant observation and (ii) the cognitive mechanisms that actually generate their actions and practices (pp. 11, 215-216). Hence, social scientists, according to Bloch, need cognitive sciences to make a distinction between (i) and (ii) and, 
thereby, to question their own and their research subjects' folk assumptions about cognition (pp. 11, 107-116).

Bloch (2012, Chapters 7-8) strengthens these points by indicating that cognitive scientists have found that many action-related cognitive processes are unconscious, automatic and fast. This means that the research subjects of social scientists cannot be aware of these processes nor of their influence on their actions and interactions. Although he grants that some traditions in the social sciences, such as the socalled "practice theories" of Pierre Bourdieu and Anthony Giddens, recognize the significance of implicit cognitive processes for social interactions, Bloch $(2012,149-154)$ maintains that the cognitive sciences are needed to analyze these processes empirically and to constrain social scientists assumptions about them. This point links his argument to Omar Lizardo and Stephen Turner's debate about whether assumptions about implicit cognitive processes in practice theories are compatible with recent findings in cognitive neuroscience (see Lizardo, 2007; Turner, 2007).

Despite his criticisms of the background assumptions of social scientists from the viewpoint of the cognitive sciences, Bloch's (2012) aim is not to completely alter the research practices and methods of the social sciences. Rather, he draws on cognitive sciences in order to constrain the cognitive assumptions of social scientists that underlie the way in which they interpret their data and formulate their research questions. He also emphasizes that the ethnographic studies of anthropologists produce such data about social phenomena that cannot be produced by means of experimental studies of cognitive scientists. Hence, not only should social scientists' assumptions about cognitive phenomena be constrained by the results of the cognitive sciences but also cognitive scientists' assumptions about social phenomena should be constrained by the robust results of the social sciences. Although Bloch's book is not targeted to cognitive scientists, the idea of mutual constraints between the cognitive and the social sciences is included in his arguments. In what follows, we focus on his ideas as to how the cognitive sciences should be taken into account in the social sciences.

Bloch's argument from constraints can be reconstructed as follows:

1) Since all social processes involve cognitive aspects, social scientists must make assumptions about human cognition in their research practices. 
2) Social scientists' assumptions about the cognitive processes of their research subjects are often based on the subjects' own accounts of these processes and/or the ideas and concepts of "folk psychology" that people use in their everyday life.

3) Cognitive scientific studies have convincingly demonstrated that our cognitive processes are not transparent to us and that our own understanding of these processes, including social scientists' and their research subjects "folk psychological theories", is limited and sometimes misleading.

4) Conclusion: social scientists assumptions about cognitive processes of their research subjects should be constrained by the results of cognitive sciences.

This argument includes much less ontological, methodological and theoretical presuppositions than the argument from explanatory grounding and the argument from theoretical unification. For example, instead of celebrating the progress of the cognitive sciences, Bloch $(2012$, p. 9) holds that "the study of cognition is in its infancy" and that, for this reason, "the cognitive sciences are more certain when telling us what things are not like, than when telling us how things are" (p. 9). Accordingly, the main purpose of Bloch's argument is to weed out implausible cognitive assumptions from the social sciences rather than to ground the social sciences in the cognitive sciences or to unify the social sciences with the help of the cognitive sciences.

We think that all of the premises of the above argument are well justified. Indeed, cognitive scientists have convincingly demonstrated not only that our everyday conceptions about how our minds work are seriously limited and potentially misleading but also that a large part of our action-related cognitive processes are implicit (e.g. Evans \& Frankish, 2009; Kahneman, 2011). We also agree with the conclusion 4 to the extent that social scientists studying small-scale social interactions are well-advised to pay attention to the results of cognitive sciences when they make assumptions about the cognitive processes of their research subjects since this enables them to avoid biased explanations. This does not mean, however, that social scientists should replace their methods with the methods of cognitive sciences, since, as Bloch (2012) rightly argues, ethnographic methods can be used to produce such data about social and cultural phenomena that is impossible to obtain by using the experimental and simulation methods of cognitive scientists (see also Hutchins, 1995). What it does mean is that the data social scientists produce by using ethnographic methods should not be interpreted as providing reliable 
knowledge about the internal cognitive processes of their research subjects and that, for many explanatory purposes, it should be supplemented with data acquired by using other type of methods.

Nevertheless, it can be argued that the results of cognitive sciences are less significant when it comes to explanatory studies on the outcomes of social interactions of a large number of individuals in a specific institutional context. For example, a defender of a rational choice theory might argue that abstractions and idealizations regarding the cognitive processes of individuals are needed to model those social mechanisms that generate the aggregate outcomes of a large number of interacting individuals in the context of markets, representative democracies or the systems of higher education. The reason is that the "cognitively realist" models of these complex interactions tend to become either intractable or so complex that it would be impossible to understand them. In addition, it has been argued that "the institutional scaffolds" that are external to individuals but constraint their choices may be the most important explanatory factors in cases of this kind, meaning that the explanatory power of rational choice models may ultimately reside in these institutional scaffolds rather than in the assumptions about those cognitive or neural processes that are internal to individuals (Clark, 1997, pp. 181-184; cf. Gintis, 2007a).

Although this is not right place to evaluate the vices and virtues of rational choice theory, or its different versions and interpretations, we contend that social scientists cannot escape from making trade-offs between the psychological realism and the tractability of their models in this context. The feasibility of their assumptions about cognition should be judged in a case-by-case manner that takes into account the purposes in which they use their models. However, in order to be able make judgements of this kind, social scientists should be aware of the relevant cognitive processes that they abstract from or idealize in their models. To this end, they need cognitive sciences (for an insightful discussion of these topics, see Lizardo, 2009).

Similar arguments that highlight the constraints that the robust findings of cognitive sciences should impose on social scientists' background assumptions about cognition and culture have been developed, among others, by Paul DiMaggio (1997), Mark Turner (2001), Karen Cerulo (2009) and Lizardo, Sepulvado, Stoltz \& Taylor (2019). All of them review theories and research results of the cognitive sciences and show how they have been (or should be) taken into account by social scientists in different 
research fields and how they can be used to formulate new research questions. In their view, the influence of the cognitive sciences on the social sciences is mediated via empirical studies on specific social phenomena. These studies might also provide new avenues for developing research programs that would aim at deeper integration of the findings, methods and theories of these disciplines.

\section{Argument from complementarity}

The idea of complementarity can be understood as an epistemological principle according to which a single perspective cannot provide comprehensive descriptions and explanations of the specific domain of phenomena. A classic example of the complementarity principle is Niels Bohr's claim that objects studied in physics include both particle and wave aspects that cannot be measured at the same time nor represented by using a single theoretical model. In this section, we address the argument according to which cognitive science and cognitive sociology are complementary perspectives. It is presented by Eviatar Zerubavel in his book Social Mindscapes: An Invitation to Cognitive Sociology that was published in 1997.

Zerubavel's (1997, Chapter 1) key argument is that cognitive science should be complemented with a perspective that falls between two extremes: cognitive universalism and cognitive individualism. The expression "cognitive universalism" characterizes the perspective of cognitive science which aims to uncover the universal attributes of human cognition (e.g. How human memory works? How many pieces of information can be held in short-term memory?). The expression "cognitive individualism" characterizes a perspective that considers every individual mind to be unique. It is difficult to find a scientific illustration of cognitive individualism. Zerubavel himself suggests psychoanalysis, but cognitive individualism could be better understood as a kind of folk understanding of human mind. The additional perspective between these extremes is cognitive sociology. Zerubavel uses the expression "sociomental" as a shorthand for the aspects of cognition that are studied in this complementary perspective.

As a rough definition, cognitive phenomena that are attributed to a certain group or culture can be regarded as sociomental (Zerubavel, 1993, p. 398). For example, memory has sociomental aspects, such as the differences how different groups remember the same event from nation's history (Zerubavel, 1997, Chapter 6). Also socially shared interpretive frameworks and social norms that constrain 
perception and focus attention belong to the domain of sociomental - e.g. how social scientists look at the world or how different groups look at abstract art (Zerubavel, 1997, Chapters 2-3). Although it seems that the boundaries of cognitive sociology that studies sociomental phenomena are quite fuzzy, the core feature of this perspective is that it "complements the work of cognitive scientists by showing that we think not only as human beings but also as social beings located in particular cultures and subcultures, and belonging to particular 'thought communities." (Brekhus, 2007, p. 450; see also Zerubavel, 1997, 5).

Cognitive sociology is historically rooted in the sociology of knowledge. Especially Simmel, Mannheim, Goffman, Berger and Luckmann are sociologists whose work has influenced on cognitive sociology (Brekhus, 2007, pp. 449-542). Therefore, Zerubavel's version of cognitive sociology also has close relationship with the tradition of social constructionism. Although his way of understanding cognitive sociology could result in adversary between cognitive science and cognitive sociology - or naturalism and social constructionism - Zerubavel aims to avoid it by considering the relationship between these disciplines as epistemically symmetrical, in the sense that none of the disciplines studying the different aspects of human cognition should be privileged over others.

Zerubavel suggests that cognitive sociology is important for the multilevel study of mind since "[i]n focusing specifically on the third, intermediate level, cognitive sociology helps widen [the limited scope of cognitive universalism and individualism] as well as avoid the reductive tendencies normally associated with those two extremes" (Zerubavel, 1997, p. 113). Hence, it serves as a critique of "reductionism" that is sometimes connected to cognitive universalism. Cognitive sociology also has a constructive side since Zerubavel $(1997$, p. 113) considers it as a contribution to the development of "an integrative, multilevel approach to cognition that views us both as individuals, as human beings, and as social beings."

In addition to its focus on cognitive variation, cognitive sociology aims to complement cognitive science by focusing on the moral and political aspects of cognition and by utilizing qualitative/interpretative methods (e.g. Brekhus, 2015). For example, the political aspects of cognition are involved when some artist is classified to be lowbrow in order to sustain prevailing hierarchies in the art world, or when there are different ways how a certain moment in some nation's history is remembered that each serve 
particular interests. Cognitive sociologists study these and other social aspects of cognitive processes by using mostly qualitative/interpretative methods, such as interviewing and document analysis (e.g. Vaughan, 2002) as well as ethnography (e.g. Brekhus, 2003).

This is our reconstruction of Zerubavel's argument from complementarity:

1) Since cognitive science studies cognitive universals, it cannot answer questions about how cognition varies between groups and how social environments affect cognitive processes.

2) In order to provide a more comprehensive understanding of human cognition, cognitive science should be complemented with studies that answer questions concerning the domain of sociomental.

3) Cognitive sociology's ontological, theoretical and methodological position allows it to answer questions concerning the domain of sociomental.

4) Conclusion: Cognitive science should be complemented with cognitive sociology.

The argument from complementarity is based on a view that human cognition is an object that is studied by many disciplines that produce knowledge according to their distinct ontological and epistemological commitments. The immediate goal is not to integrate (in a strong sense) these disciplines but rather to produce complementary knowledge about cognition. Hence, although the shared object of study in these disciplines is human cognition, the knowledge is always tied to the discipline which produced it. The relationship between different disciplines may be described as epistemically symmetrical: they all have the same object of study, but none of them have privilege over it.

The argument from complementarity may be quite compelling to some social scientists since premises 2 and 3 claim that cognitive sociology has unique perspective for studying human cognition and should be therefore included in the group of disciplines that study cognition. If social scientists are worried about the identity of their discipline, this argument can be used to strengthen this identity, since it claims that without social scientific perspective the study of cognition remains incomplete. Nevertheless, more significant challenge to the argument from complementarity is that it needs to convince cognitive scientists for the need of cognitive sociology. It is not at all certain that is able to do that. 
One problem with this argument that is reflected in premise 1 is that it is based on a quite narrow understanding of the cognitive sciences. It seems to ignore how cognitive sciences have moved away from a nearly exclusive focus on "the universal foundations of human cognition" (Zerubavel, 1997, p. 3), and included wider perspectives on human cognition that focus on the embodied, embedded, enactive, materially extended, situated, socially distributed and cultural-historical aspects of cognitive processes (e.g. Hutchins, 1995; Clark, 1997; Franks, 2011; Lizardo et al., 2019; Milkowski et al., 2018; Turner, 2018). Although studies on "wide cognition" were in their infancy in 1990's, when Zerubavel first developed his argument from complementarity, it seems to us that these wider perspectives tend to be ignored in more recent discussion in Zerubavellian cognitive sociology as well (e.g. Brekhus, 2015). Hence, we suggest that the argument from complementarity needs to be updated by taking into account of these new perspectives. When this is done, it is not at all clear whether the revised argument can be regarded as a distinct type of argument from the other arguments for the cognitive social sciences that we have analyzed above.

Other problem with the argument from complementarity concerns the kind of interdisciplinarity it would produce in practice. Omar Lizardo (2014), for example, argues that the sociology of culture and cognition, often used as a synonym of cognitive sociology, creates "a sense of pseudointerdisciplinarity". This means that, although the name suggests at least some degree of interdisciplinary interaction, there actually is minimal communication between the disciplines. It seems to us that all attempts to create complementary perspectives to cognitive science are at risk of falling into the trap of pseudo-interdisciplinarity of this kind. Hence, although interdisciplinary integration is regarded as an ultimate goal of the multilevel approach to cognition in some of Zerubavel's (e.g. 1997, p. 113) claims, we may ask if the argument from complementary actually leads away from this goal.

\section{Discussion and conclusions}

To recapitulate the main points of the above discussions, we have analyzed four different types of arguments for integrating the social sciences with the cognitive sciences: the argument from explanatory grounding, the argument from theoretical unification, the argument from constraints, and the argument from complementarity. The argument from explanatory grounding was exemplified by the reasons that Ron Sun provides for using cognitive scientific knowledge as a basis for building 
explanations for social phenomena. The argument from theoretical unification was illustrated by Herbert Gintis's proposal to unify behavioral science with the revised versions of the rational actor model and game theory. The argument from constraints was exemplified by Maurice Bloch's views according to which social scientists must make assumptions about the cognition of their research subjects, and these assumptions ought to be constrained by the findings of the cognitive sciences. And the argument from complementarity was exemplified by Eviatar Zerubavel's approach to cognitive sociology which studies variation in cognition between groups and cultures in a way that is taken to complement the traditional cognitive science. We organized the arguments in sequence such that the argument from explanatory grounding (which we discussed first) is the strongest form of interdisciplinary integration between the cognitive and the social sciences, while the argument from complementarity (which we discussed last) is the weakest one. In what follows, we briefly compare these arguments by analyzing their presuppositions concerning how social phenomena can be made understandable in scientific terms.

The argument from explanatory grounding can be naturally combined with the mechanistic approach to scientific explanation. The fundamental insight underlying the mechanistic approach is that many "special sciences" (e.g. the biosciences, the cognitive sciences and at least some parts of the social sciences) track causal mechanisms that produce, underlie, enable or maintain specific types of empirical phenomena (e.g. Bechtel, 2008; Craver \& Darden, 2013). Accordingly, the argument from explanatory grounding claims that social sciences should be grounded in the cognitive sciences because the latter provide knowledge about those cognitive mechanisms that underlie and enable social phenomena. Although the argument from explanatory grounding was exemplified with Sun's CLARION architecture, the mechanistic approach to explanation does not require that those cognitive mechanisms that underlie and enable social phenomena have to be universal and modular. Nor does it require that all cognitive mechanisms have to be internal to individuals (e.g. Miłkowski et al., 2018).

The argument from theoretical unification presupposes the unification approach to explanation. The traditional version of this approach maintains that "science increases our understanding of the world by reducing the total number of independent phenomena that we have to accept as ultimate or given" (Friedman, 1974, 15). The unification approach to explanation does not require that unifying explanations of social phenomena should be provided in terms of cognitive mechanisms. Rather, it 
suggests that we need a unifying theoretical framework that can be used to explain both social and cognitive phenomena. This is precisely what Gintis aims to achieve by his updated versions of the rational actor model and game theory that he provides as a unified modeling framework for the behavioral sciences, including the overlapping areas of the cognitive and social sciences. In other words, the unification approach implies a "top-down" view of interdisciplinary integration since it emphasizes the need to fit phenomena studied in different disciplines within a general theoretical framework in order to understand them while the mechanistic approach is more "bottom-up" view due to the fact that it highlights the need to study causal mechanisms that underlie and enable specific types of phenomena in order to understand them (cf. Kitcher, 1985). Although some advocates of the unification approach consider it as an explication of causal explanations, we think that many explanations that may be said to theoretically unify phenomena are not causal explanations. For example, explanations in terms of game theoretical equilibria do not explicate any causal processes or mechanisms. ${ }^{6}$

One way to understand the argument from complementarity is to make a distinction between causal explanations and interpretations of cognitive phenomena. Insofar as cognitive science provides causal explanations for cognitive phenomena in terms of universal properties and mechanisms, then cognitive sociology in Zerubavel's sense seeks to complement cognitive science by providing interpretive accounts of those cognitive phenomena that vary between different groups and cultures. Hence, the focus in cognitive sociology seems to be on explicating the socially shared meanings of cognitive phenomena in different cultures and groups rather than on identifying the cognitive causal mechanisms that contribute to social phenomena. This also appears to be the reason why cognitive sociologists rely on case study designs and qualitative methods in their empirical studies. The interpretive emphasis of the argument from complementarity clearly separates it from the above arguments which both emphasize the need to provide scientific explanations for social phenomena, not just interpretations of them.

Unlike these three arguments, the argument from constraints does not presuppose a specific approach to scientific explanation or interpretation. It is also more interactive than any of the other arguments.

\footnotetext{
${ }^{6}$ The mechanistic and unification approaches to explanation also seem to presuppose a different concept of causation but we do not address this issue here.
} 
The argument acknowledges not only that cognitive sciences should constrain social scientists' assumptions about cognitive phenomena but also that social sciences should constrain cognitive scientists' assumptions about social phenomena. Although Bloch nearly exclusively focuses on the influence of the cognitive sciences on the social sciences, the interdisciplinary traffic to the other direction is implied in his argument. In other words, cognitive scientists' assumptions about social phenomena should also be constrained by the robust findings of the social sciences since cognitive scientists tend to lack the proper theoretical frameworks and empirical methods to make sense of social phenomena.

In our view, the argument from explanatory grounding and the argument from constraints make the most compelling cases for the cognitive social sciences. These two arguments are genuinely integrative in the sense that they indicate promising ways in which the cognitive and social sciences can be brought together. Although the argument from constraints can be seen as to offer guidelines towards the explanatory grounding of social phenomena in the knowledge provided by the cognitive sciences, there are good reasons, as was shown above, to re-interpret the latter argument in a piecemeal manner that encourages two-way traffic between these two types of disciplines. In contrast to these two arguments, the argument from theoretical unification is able to unify the cognitive and social sciences only at the expense of large portions of both of these sciences being either rejected or ignored. We do not find this type of grand theoretical unification a feasible goal for the cognitive social sciences although we are open to the possibility that the cognitive social sciences may locally unify some social phenomena that are studied in different research fields of the current social sciences. Also the argument from complementarity seems counterproductive to the cognitive social sciences since it defends prevailing disciplinary boundaries rather than provides reasons for crossing them.

Finally, we think that Sun's version of the argument from explanatory grounding needs to be revised in two respects. First, a more externalist (or "wide") understanding of cognition than that assumed in Sun's argument is needed in order to provide explanatory grounding for many social phenomena that are studied in the social sciences since these phenomena involve cognitive processes that have social or cultural aspects. Although there is no consensus about the definition of cognition among the advocates of the externalist approaches in cognitive science-such as those developed in 4E cognition 
approaches $^{7}$ as well as situated and distributed cognition approaches-, it is clear that this growing family of externalist approaches challenges what Stephen Turner (2018, 45-51) calls "the standard approach" to cognitive science. The standard approach is internalist in the sense that it requires that all cognitive processes are realized in the brain (or in the central nervous system) and relies on some version of the brain-computer metaphor. However, it remains to be seen to what extent these externalist approaches are able to transform cognitive sciences in a way that makes social and cultural aspects of cognition more prominent in the mainstream cognitive sciences (for an interesting discussion of the various issues involved in this debate, see Turner, 2018).

Second, we need a context-sensitive version of the mechanistic approach to explanation in order to explicate the explanatory grounding relations between the social and cognitive sciences. Although the mechanistic approach has emphasized the multilevel nature of mechanism-based explanations in the life sciences (e.g. Craver \& Darden, 2013), when this model of explanation has been applied in the cognitive and social sciences, there has been a tendency to focus mostly on underlying mechanisms that have been assumed to provide the micro-foundations for higher level cognitive and social phenomena (Bechtel, 2009; Kaidesoja, 2013). Even though we do not deny the importance of studying the micro-foundations of cognitive and social phenomena in terms of their underlying mechanisms, in many cases the broader contexts of these mechanisms, including the higher level mechanisms that affect the operations of their component mechanisms, are equally important when it comes to providing mechanistic explanations to cognitive and social phenomena (e.g. Bechtel, 2009; Kaidesoja, 2013; Miłkowski et al., 2018). This is especially the case when we are interested in cognitive phenomena that involve important social and cultural aspects which are highlighted by externalist approaches to cognition. Our next task is to develop this kind of mechanistic approach for the cognitive social sciences. ${ }^{8}$

\section{References}

\footnotetext{
74 E cognition refers to the embodied, embedded, enactive and extended approaches to cognition (see Newen, De Bruin \& Gallagher, 2018).

${ }^{8}$ We would like to thank Ronny Puustinen for his valuable comments on the earlier draft of this paper. Kaidesoja would like to thank Kone Foundation for research funding. Sarkia and Hyyryläinen would like to thank The Emil Aaltonen Foundation for research funding.
} 
Barkow, J. H., Cosmides, L., \& Tooby, J. (1992). The adapted mind: Evolutionary psychology and the generation of culture. Oxford: Oxford University Press.

Bechtel, W. (2008). Mental mechanisms: Philosophical perspectives on cognitive neuroscience. London: Routledge.

Bechtel, W. (2009). Looking down, around, and up: Mechanistic explanation in psychology. Philosophical Psychology, 22(5), 543-564.

Bloch, M. (2012). Anthropology and the cognitive challenge. Cambridge: Cambridge University Press.

Boyer, P. (2001). Religion explained: The evolutionary origins of religious thought. New York: Basic Books.

Brekhus, W. (2015). Culture and cognition: Patterns in the social construction of reality. Cambridge: Polity Press.

Brekhus, W. (2003). Peacocks, chameleons, centaurs: Gay suburbia and the grammar of social identity. Chicago: Chicago University Press.

Brekhus, W. (2007). The Rutgers School: A Zerubavelian culturalist cognitive sociology. European Journal of Social Theory, 10(3), 448-464.

Buller, D. (2005). Adapting minds: Evolutionary psychology and the persistent quest for human nature. Cambridge, MA: The MIT Press.

Cartwright, N. (1999). The dappled world: A study of the boundaries of science. Cambridge: Cambridge University Press.

Cerulo, K. A. (2009). Mining the intersections of cognitive sociology and neuroscience. Poetics, 38(2), $115-132$.

Clark, A. (1997). Being there: Putting brain, body, and world together again. Cambridge, MA: The MIT Press.

Craver, C.F., \& Darden L. (2013). In search of mechanisms: Discoveries in the life sciences. Chicago: The University of Chicago Press. 
DiMaggio, P. (1997). Culture and cognition. Annual Review of Sociology, 23(1), 263-287.

Donald, M. (2001). A mind so rare: The evolution of human consciousness. New York and London: W.W. Norton \& Company.

Dupré, J. (1993). The disorder of things: Metaphysical foundations for the disunity of science. Cambridge, MA: Harvard University Press.

Dupré, J. (2001). Human nature and the limits of science. Oxford: Oxford University Press.

Edwards, D., \& Potter J. (1992). Discursive psychology. London: Sage.

Evans, J., \& Frankish K. (Eds.). (2009). In two minds: Dual process theories and beyond. Oxford: Oxford University Press.

Franks, B. (2011). Culture \& cognition: Evolutionary perspectives. Houndmills: Palgrave Macmillan.

Friedman, M. (1974). Explanation and scientific understanding. Journal of Philosophy, 71, 5-19.

Fumagalli, R. (2013). The futile search for true utility. Economics \& Philosophy, 29, 325-347.

Gintis, H. (2004). Towards the unity of the behavioral sciences. Politics, Philosophy \& Economics, 3, 3757.

Gintis, H. (2007a). A framework for the unification of the behavioral sciences. Behavioral and Brain Sciences, 30, 1-16.

Gintis, H. (2007b). A framework for the unification of the behavioral sciences II. Behavioral and Brain Sciences, 30, 45-53.

Gintis, H. (2009). The bounds of reason: Game theory and the unification of the behavioral sciences. Princeton, NJ: Princeton University Press.

Gintis, H. (2012). The role of cognitive processes in unifying the behavioral sciences. In R. Sun (Ed.), Grounding social sciences in cognitive sciences (pp. 415-443). Cambridge, MA: MIT Press.

Hechter, M., \& Kanazawa, S. (1997). Sociological rational choice theory. Annual Review of Sociology, 23, 191-214. 
Hutchins, E. (1995). Cognition in the wild. Cambridge, MA: The MIT Press.

Ignatow, G. (2014). Ontology and method in cognitive sociology. Sociological Forum, 29(4), 990-994.

Kahneman, D. (2011). Thinking, fast and slow. London: Penguin Books.

Kaidesoja, T. (2013). Overcoming the biases of microfoundationalism: Social mechanisms and collective agents. Philosophy of the Social Sciences, 43(3), 301-322.

Karmiloff-Smith, A. (2015). An alternative to domain-general or domain-specific frameworks for theorizing about human evolution and ontogenesis. AIMS Neurosci., 2(2), 91-104

Kim, J. (2005). Physicalism, or something near enough. Princeton, NJ: Princeton University Press.

Kitcher, P. (1985). Two approaches to explanation. Journal of Philosophy, 82, 632-639.

Lizardo, O. (2007). "Mirror neurons," collective objects and the problem of transmission: Reconsidering Stephen Turner's critique of practice theory. Journal for the Theory of Social Behaviour, 37(3), 319-350.

Lizardo, O. (2009). Formalism, behavioral realism and the interdisciplinary challenge in sociological Theory. Journal for the Theory of Social Behaviour, 39(1), 39-79.

Lizardo, O. (2014). Beyond the Comtean schema: The sociology of culture and cognition versus cognitive social science. Sociological Forum, 29(4), 983-989.

Lizardo, O., Sepulvado, B., Stoltz, D., \& Taylor, M.A. (2019) What can cognitive neuroscience do for cultural sociology? American Journal of Cultural Sociology. Retrieved August 6, 2019, from https://doi.org/10.1057/s41290-019-00077-8.

Longino, H. (1990). Science as social Knowledge. Princeton, NJ: Princeton University Press.

Longino, H. (2013). Studying human behavior: How scientists investigate aggression and sexuality. Chicago: The University of Chicago Press.

Mäki, U. (2009). Economics imperialism: Concepts and constraints. Philosophy of the Social Sciences 39 (3): 351-380. 
Milkowski, M., Clowes, R., Rucińska, Z., Przegalińska, A., Zawidzki, T., Krueger, J., ... Hohol, M. (2018). From wide cognition to mechanisms: A silent revolution. Frontiers of Psychology 9, Art. 2393.

Newen, A., De Bruin, L., \& Gallagher, S. (Eds.) (2018) The Oxford handbook of 4E cognition. Oxford: Oxford University Press.

Pinker, S. (2002). The blank slate: The modern denial of human nature. London: Penguin Books.

Sperber, D. (1996). Explaining culture: A naturalist approach. Oxford: Blackwell Publishers.

Sterelny, K. (2003). Thought in a hostile world: The evolution of human cognition. Malden, MA: Blackwell Publishing.

Sterelny, K. (2012). The evolved apprentice. Cambridge, MA: MIT Press.

Sun, R. (2012). Prolegomena to cognitive social sciences. In R. Sun (Ed.), Grounding social sciences in cognitive sciences (pp. 3-32). Cambridge, MA: MIT Press.

Sun, R. (2017). The CLARION cognitive architecture: Toward a comprehensive theory of the mind. In S.E.F. Chipman (Ed.), The Oxford handbook of cognitive science (pp. 117-135). Oxford: Oxford University Press.

Turner, M. (2001). Cognitive dimensions of social science. Oxford: Oxford University Press.

Turner, S.P. (2007). Mirror neurons and practices: A response to Lizardo. Journal for the Theory of Social Behaviour 37(3), 351-371.

Turner, S.P. (2018). Cognitive science and the social. London: Routledge.

Vaughan, D. (2002). Signals and interpretive work: The role of culture in a theory of practical action. In K.A. Cerulo (Ed.), Culture in mind: Toward a sociology of culture and cognition (pp. 28-54). London: Routledge.

Weisberg, M., \& Muldoon, R. (2009). Epistemic landscapes and the division of cognitive labor. Philosophy of Science, 76(2), 225-252.

Wimsatt, W.C. (1997). Aggregativity: Reductive heuristics for finding emergence. Philosophy of Science, 64, 371-384. 
Zerubavel, E. (1993). Horizons: On the sociomental foundations of relevance. Social Research, 60(2), 397-413.

Zerubavel, E. (1997). Social Mindscapes: An Invitation to cognitive sociology. Cambridge, MA: Harvard University Press. 